\title{
Totally dissipative systems
}

\author{
O. Kaneko ${ }^{\mathrm{a}, *}$, P. Rapisarda ${ }^{\text {b,1 }}$, K. Takaba ${ }^{\mathrm{c}, 2}$ \\ ${ }^{a}$ Graduate School of Engineering Science, Osaka University, 1-3 Machikaneya-mamachi, Toyonaka, Osaka 560-8531, Japan \\ ${ }^{\mathrm{b}}$ Department of Mathematics, University of Maastricht, P.O. Box 616, 6200 MD Maastricht, The Netherlands \\ ${ }^{\mathrm{c}}$ Department of Applied Mathematics and Physics, Graduate School of Informatics, Kyoto University, Kyoto 606-8501, Japan
}

Received 14 November 2003; received in revised form 12 November 2004; accepted 14 November 2004

Available online 25 December 2004

\begin{abstract}
In a totally dissipative behavior, all non-trivial trajectories dissipate energy. A characterization of such behaviors is given in terms of properties of the one- and two-polynomial matrices associated with the supply rate and with their kernel- and image representation.
\end{abstract}

(C) 2004 Elsevier B.V. All rights reserved.

Keywords: Two-variable polynomial matrices; Quadratic difference forms; Dissipative systems; Behavioral approach

\section{Introduction}

In this paper, we study dissipative systems in the framework originated in $[7,8]$ and further developed in $[1,2,6,11]$. In such framework, a dissipative dynamical system is one for which two functions are specified: one is a supply rate function measuring the rate at which energy flows into the system, and the other is a storage function measuring the amount of energy stored into the system. The two functions are related by the dissipation inequality, which expresses the

\footnotetext{
* Corresponding author. Tel.: +8168506341.

E-mail addresses: kaneko@ft-lab.sys.es.osaka-u.ac.jp (O. Kaneko), P.Rapisarda@math.unimaas.nl (P. Rapisarda), takaba@amp.i.kyoto-u.ac.jp (K. Takaba).

${ }^{1}$ Tel.: +31433883360; fax: +31433211889.

${ }^{2}$ Fax: +0757535507 .
}

intuitive property that in a dissipative system the storage of energy cannot happen faster than the speed with which energy is supplied to it from the environment. The difference between the supplied and the stored energy is the dissipated energy, which is measured by a nonnegative functional called the dissipation rate.

In this paper, we consider a special class of discretetime dissipative systems, that consisting of those systems for which the dissipation rate is instantaneously positive on all nonzero trajectories of the system. We call such systems totally dissipative, because in such cases, the transfer of the state from one point of the state space to another cannot be performed without dissipation. The main result of the paper is a theorem characterizing such class of systems in terms of properties of their trajectories and of properties of the polynomial matrices corresponding to their representation. 
Notation. In this paper we denote the sets of real numbers with $\mathbb{R}$ and the set of integers with $\mathbb{Z}$. The space of $n$ dimensional real vectors is denoted by $\mathbb{R}^{\mathrm{n}}$, and the space of $\mathrm{m} \times \mathrm{n}$ real matrices, by $\mathbb{R}^{\mathrm{m} \times \mathrm{n}}$. If $A \in$ $\mathbb{R}^{\mathrm{m} \times \mathrm{n}}$, then $A^{\mathrm{T}} \in \mathbb{R}^{\mathrm{n} \times \mathrm{m}}$ denotes its transpose. Whenever the size of a matrix or a vector is not specified, a bullet $\bullet$ is used. In order to enhance readability, when dealing with a vector space $\mathbb{R}^{\bullet}$ whose elements are commonly denoted with $w$, we use the notation $\mathbb{R}^{\mathrm{w}}$; similar considerations hold for matrices representing linear operators on such spaces. Given two matrices $A$ and $B$ with the same number of columns, we denote with $\operatorname{col}(A, B)$ the matrix obtained by stacking $A$ over B. If $A_{i} \in \mathbb{R}^{\mathrm{k}_{i} \times \mathrm{k}_{i}}, i=1, \ldots, \mathrm{m}$, then $\operatorname{diag}\left(A_{i}\right)_{i=1, \ldots, \mathrm{m}}$ denotes the $\left(\sum_{i=1}^{\mathrm{m}} \mathrm{k}_{\mathrm{i}}\right) \times\left(\sum_{i=1}^{\mathrm{m}} \mathrm{k}_{\mathrm{i}}\right)$ matrix having the $A_{i}$ 's on the main diagonal.

The set consisting of all sequences from $\mathbb{Z}$ to $\mathbb{R}^{\mathrm{W}}$ is denoted with $\left(\mathbb{R}^{\mathrm{w}}\right)^{\mathbb{Z}}$. On such space we define the left, i.e. backwards, shift

$\sigma:\left(\mathbb{R}^{\mathrm{w}}\right)^{\mathbb{Z}} \rightarrow\left(\mathbb{R}^{\mathrm{w}}\right)^{\mathbb{Z}}$

$(\sigma w)(t):=w(t+1)$

for all $t \in \mathbb{Z}$. The set of square-summable w-dimensional vector time series is denoted with $l_{2}\left(\mathbb{Z}, \mathbb{R}^{\mathrm{W}}\right)$, i.e. $w \in l_{2}\left(\mathbb{Z}, \mathbb{R}^{\mathrm{W}}\right)$ if $\sum_{k=-\infty}^{\infty} w^{\mathrm{T}}(k) w(k)<\infty$.

The ring of polynomials with real coefficients in the indeterminate $\xi$ is denoted by $\mathbb{R}[\xi]$; the ring of twovariable polynomials with real coefficients in the indeterminates $\zeta$ and $\eta$ is denoted by $\mathbb{R}[\zeta, \eta]$. Similarly, the ring of "polynomials" (or finite Laurent series) with both positive and negative powers of $\xi$ is denoted with $\mathbb{R}\left[\xi^{-1}, \xi\right]$. The space of all $\mathrm{n} \times \mathrm{m}$ polynomial matrices in the indeterminate $\xi$ is denoted by $\mathbb{R}^{\mathrm{n} \times \mathrm{m}}[\xi]$, and that consisting of all $\mathrm{n} \times \mathrm{m}$ polynomial matrices in the indeterminates $\zeta$ and $\eta$ by $\mathbb{R}^{\mathrm{n} \times \mathrm{m}}[\zeta, \eta]$. Given a polynomial matrix $R(\xi):=R_{0}+R_{1} \xi+\cdots+R_{L-1} \xi^{L-1}+R_{L} \xi^{L} \in$ $\mathbb{R}^{\mathrm{n} \times \mathrm{m}}[\xi]$ with $R_{L} \neq 0$, we define its reciprocal matrix $R^{r}(\xi)$ as $R^{r}(\xi):=R_{0} \xi^{L}+R_{1} \xi^{L-1}+\cdots+R_{L-1} \xi+$ $R_{L} \in \mathbb{R}^{\mathrm{n} \times \mathrm{m}}[\xi]$. A matrix $U \in \mathbb{R}\left[\xi^{-1}, \xi\right]$ is called unimodular if its inverse also belongs to $\mathbb{R}\left[\xi, \xi^{-1}\right]$. It is easy to see that this is equivalent with $\operatorname{det}(U)=\alpha \xi^{i}$ for some nonzero $\alpha \in \mathbb{R}$ and for some $i \in \mathbb{Z}$.

\section{Linear difference systems}

In this paper, we consider linear, shift-invariant with time axis $\mathbb{Z}$, and closed subspaces of $\left(\mathbb{R}^{\mathrm{W}}\right)^{\mathbb{Z}}$ equipped with the topology of pointwise convergence. We denote the set of such behaviors with $\mathscr{L}^{\mathrm{w}}$. It has been shown in [4,9] that if $\mathfrak{B} \in \mathscr{L}^{\mathrm{w}}$, then there exists a polynomial matrix $R \in \mathbb{R}^{\bullet \times \mathrm{W}}[\xi]$ such that

$\mathfrak{B}=\left\{w \in\left(\mathbb{R}^{\mathrm{W}}\right)^{\mathbb{Z}} \mid R(\sigma) w=0\right\}$,

where, if $R=R_{0}+R_{1} \xi+\cdots+R_{L} \xi^{L}$, with $R_{i} \in \mathbb{R}^{\bullet \times \mathrm{w}}$, then

$R(\sigma) w:=R_{0} w+R_{1}(\sigma w)+\cdots+R_{L}\left(\sigma^{L} w\right)$.

Moreover, we can always take the number of rows of $R$ to be less than or equal to w. The representation of $\mathfrak{B}$ as the kernel of the polynomial shift operator $R(\sigma)$ is called a kernel representation of $\mathfrak{B}$. Such representation is called minimal if $R$ has the minimal number of rows in any kernel representation of $\mathfrak{B}$.

Some behaviors can also be represented as the image of polynomial operators in the shift. Let $M \in$ $\mathbb{R}^{\mathrm{w} \times} \cdot[\xi]$, and consider the linear subspace of $\mathscr{L}^{\mathrm{w}}$ defined by

$\mathfrak{B}:=\left\{w \in\left(\mathbb{R}^{\mathrm{w}}\right)^{\mathbb{Z}} \mid \exists \ell \in\left(\mathbb{R}^{\bullet}\right)^{\mathbb{Z}}\right.$

such that $w=M(\sigma) \ell\}$.

Such representation is called an image representation of $\mathfrak{B}$, and $\ell$ is called the latent variable. Evidently, the equation $w=M(\sigma) \ell$ also constrains the full trajectory $\operatorname{col}(w, \ell)$; the linear subspace of $\mathscr{L}^{\mathrm{w}+1}$ defined by

$\mathfrak{B}_{\mathrm{f}}:=\left\{(w, \ell) \in\left(\mathbb{R}^{\mathrm{w}+1}\right)^{\mathbb{Z}} \mid w=M(\sigma) \ell\right\}$

is called the full behavior associated with the image representation $\mathfrak{B}=\operatorname{Im} M(\sigma)$. It can be shown that only controllable behaviors can be described as the image of some polynomial difference operator (see $[4,9]$ for details on the notion of controllability in the behavioral framework). It can be shown that two full row-, respectively column, rank matrices $R \in \mathbb{R}^{(\mathrm{W}-1) \times \mathrm{W}}$ and $M \in \mathbb{R}^{\mathrm{w} \times \mathrm{l}}$ define a kernel, respectively image, representation of the same controllable behavior if and only if $R M=0$. In an image representation $w=M(\sigma) \ell$, the latent variable $\ell$ is called observable from $w$ if $M(\sigma) \ell=0$ implies $\ell=0$. It can be shown that this is the case if and only if for every $\lambda \in \mathbb{C}$ the matrix $M(\lambda)$ has full column rank. 


\section{Quadratic difference forms}

In this section, we review the definitions and results on quadratic difference forms (cf. [1]), which is the discrete time version of quadratic differential forms developed in [11] in the continuous time, required in the rest of this paper.

Let $\Phi \in \mathbb{R}^{\mathrm{W}_{1} \times \mathrm{W}_{2}}[\zeta, \eta]$; then

$\Phi(\zeta, \eta)=\sum_{h, k=0}^{N} \Phi_{h, k} \zeta^{h} \eta^{k}$,

where $\Phi_{h, k} \in \mathbb{R}^{\mathrm{W}_{1} \times \mathrm{W}_{2}}$ and $N$ is a nonnegative integer. The two-variable polynomial matrix $\Phi$ induces the $b i$ linear difference form (in the following abbreviated with $B d F$ )

$L_{\Phi}:\left(\mathbb{R}^{\mathrm{W}_{1}}\right)^{\mathbb{Z}} \times\left(\mathbb{R}^{\mathrm{W}_{2}}\right)^{\mathbb{Z}} \longrightarrow \mathbb{R}^{\mathbb{Z}}$,

$L_{\Phi}\left(w_{1}, w_{2}\right)(t):=\sum_{h, k=0}^{N} w_{1}(t+h)^{\mathrm{T}} \Phi_{h, k} w_{2}(t+k)$.

If $\mathrm{w}_{1}=\mathrm{w}_{2}=\mathrm{w}$, then $\Phi(\zeta, \eta)$ induces a quadratic functional defined as

$Q_{\Phi}:\left(\mathbb{R}^{\mathrm{W}}\right)^{\mathbb{Z}} \longrightarrow \mathbb{R}^{\mathbb{Z}}$

$Q_{\Phi}(w)(t):=\sum_{h, k=0}^{N} w(t+h)^{\mathrm{T}} \Phi_{h, k} w(t+k)$.

Without loss of generality, we can assume the twovariable polynomial matrix $\Phi(\zeta, \eta)$ to be symmetric, i.e. $\Phi(\zeta, \eta)=\Phi(\eta, \zeta)^{\mathrm{T}}$. We call $Q_{\Phi}$ the quadratic difference form (in the following often abbreviated as $Q d F$ ) associated with the two-variable polynomial matrix $\Phi \in \mathbb{R}^{\mathrm{w} \times \mathrm{w}}[\zeta, \eta]$.

The following map:

$\partial: \mathbb{R}^{\mathrm{w} \times \mathrm{w}}[\zeta, \eta] \longrightarrow \mathbb{R}^{\mathrm{w} \times \mathrm{w}}\left[\xi^{-1}, \xi\right]$,

$\partial \Phi(\xi):=\Phi\left(\xi^{-1}, \xi\right)$

will be useful in the rest of this paper. Observe that if $\Phi \in \mathbb{R}^{\mathrm{W} \times \mathrm{W}}[\zeta, \eta]$ is symmetric, then $\partial \Phi\left(\xi^{-1}, \xi\right)=$ $(\partial \Phi)\left(\xi, \xi^{-1}\right)^{\mathrm{T}}$.

Another notion extensively used in the following is that of rate of change of a $\mathrm{QdF}$. Given a $\mathrm{QdF} Q_{\Phi}$ we define its rate of change as the $\mathrm{QdF} \nabla Q_{\Phi}$ defined by

$\nabla Q_{\Phi}(w)(t):=Q_{\Phi}(w)(t+1)-Q_{\Phi}(w)(t)$.
In terms of the two-variable polynomial matrices associated with the BdF's, the relationship between a $\mathrm{BdF}$ and its rate of change is expressed as

$\nabla \Phi(\zeta, \eta)=(\zeta \eta-1) \Phi(\zeta, \eta)$.

In this paper, we also use the series obtained from a QdF. In order to make sure that such series exist, we assume in this case that the trajectories on which the $\mathrm{QdF}$ acts are in $l_{2}\left(\mathbb{Z}, \mathbb{R}^{\mathrm{W}}\right)$. Formally, we define the series associated with $Q_{\Phi}$ as the functional $\sum Q_{\Phi}$ defined by

$$
\begin{aligned}
& \sum Q_{\Phi}: l_{2}\left(\mathbb{Z}, \mathbb{R}^{\mathrm{w}}\right) \longrightarrow \mathbb{R}, \\
& \left(\sum Q_{\Phi}\right)(w):=\sum_{t=-\infty}^{+\infty} Q_{\Phi}(w)(t) .
\end{aligned}
$$

The following is a characterization of $\sum Q_{\Phi}$ in terms of properties of the polynomial matrices $\Phi(\zeta, \eta)$ and $\partial \Phi\left(\xi^{-1}, \xi\right)$ associated with $Q_{\Phi}$.

Proposition 1. Let $\Phi \in \mathbb{R}^{\mathrm{w} \times \mathrm{w}}[\zeta, \eta]$. The following statements are equivalent:

1. $\sum Q_{\Phi}=0$;

2. There exists a $\Psi \in \mathbb{R}^{\mathrm{w} \times \mathrm{w}}[\zeta, \eta]$ such that $\nabla \Psi=\Phi$;

3. $\partial \Phi=0$.

Proof. See [1, Proposition 3.2].

Another notion extensively used in the following is that of positivity of a $\mathrm{QdF}$. We call the QdF $Q_{\Phi}$ acting on $\mathrm{w}$-dimensional time series nonnegative (denoted $\left.Q_{\Phi} \geqslant 0\right)$ if $Q_{\Phi}(w) \geqslant 0$ for all $w \in\left(\mathbb{R}^{\mathrm{W}}\right)^{\mathbb{Z}}$. We call $Q_{\Phi}$ positive (denoted $Q_{\Phi}>0$ ) if $Q_{\Phi}(w)>0$ for all $w \in\left(\mathbb{R}^{\mathrm{W}}\right)^{\mathbb{Z}}, w \neq 0$. It is not difficult to see that $Q_{\Phi} \geqslant 0$ if and only if there exists $D \in \mathbb{R}^{\bullet \times \mathrm{W}}[\xi]$ such that $\Phi(\zeta, \eta)=D^{\mathrm{T}}(\zeta) D(\eta)$. Moreover $Q_{\Phi}>0$ if and only if such $D$ satisfies $\operatorname{rank}(D(\lambda))=$ w for all $\lambda \in \mathbb{C}$.

Finally, we introduce the concept of orthogonal behaviors with respect to a given constant BdF. Let $\Phi \in$ $\mathbb{R}^{\mathrm{w} \times \mathrm{w}}$, and let $\mathfrak{B}_{1}, \mathfrak{B}_{2} \in \mathscr{L}^{\mathrm{w}}$ be controllable. Then $\mathfrak{B}_{1}$ is said to be orthogonal to $\mathfrak{B}_{2}$ with respect to $\Phi$ if

$$
\sum_{t=-\infty}^{+\infty} L_{\Phi}\left(w_{1}, w_{2}\right)(t)=0
$$

for all $w_{1} \in \mathfrak{B}_{1} \cap l_{2}\left(\mathbb{Z}, \mathbb{R}^{\mathrm{W}}\right), w_{2} \in \mathfrak{B}_{2} \cap l_{2}\left(\mathbb{Z}, \mathbb{R}^{\mathrm{W}}\right)$. Given $\mathfrak{B} \in \mathscr{L}^{\mathrm{w}}$ controllable and $\Phi \in \mathbb{R}^{\mathrm{w} \times \mathrm{w}}$, we define 
its orthogonal complement with respect to $\Phi$ to be the controllable part of the subspace defined by

$$
\begin{aligned}
& \left\{w \in\left(\mathbb{R}^{\mathrm{W}}\right)^{\mathbb{Z}} \mid \sum_{t=-\infty}^{+\infty} L_{\Phi}\left(w, w^{\prime}\right)(t)=0\right. \\
& \text { for all } \left.w^{\prime} \in \mathfrak{B} \cap l_{2}\left(\mathbb{Z}, \mathbb{R}^{\mathrm{W}}\right)\right\} .
\end{aligned}
$$

The orthogonal complement of $\mathfrak{B}$ with respect to $\Phi$ is denoted by $\mathfrak{B}^{\perp_{\Phi}}$. The following result shows how to compute a kernel or image representation of $\mathfrak{B}^{\perp_{\Phi}}$ of a discrete-time behavior $\mathfrak{B}$ with respect to a nonsingular $\Phi \in \mathbb{R}^{\mathrm{W} \times \mathrm{w}}$ (see also Section 10 of [11] for the analogous result in continuous-time).

Proposition 2. Let $\Phi \in \mathbb{R}^{\mathrm{w} \times \mathrm{w}}$ be nonsingular. Then, $w=M(\sigma) \ell$ is an observable image representation of $\mathfrak{B}$ if and only if

$M^{r}(\sigma)^{\mathrm{T}} \Phi v=0$

is a minimal kernel representation of $\mathfrak{B}^{\perp_{\Phi}}$.

Proof. Observe that $\sum_{t=-\infty}^{\infty} v(t)^{\mathrm{T}} \Phi w(t)=0$ for all $w \in \mathfrak{B}$ and all $v \in \mathfrak{B}^{\perp_{\Phi}}$ is equivalent to $\sum_{t=-\infty}^{\infty} v(t)^{\mathrm{T}} \Phi(M(\sigma) \ell)(t)=0$ for all $v \in \mathfrak{B}^{\perp_{\Phi}}$ and all $\ell \in l_{2}\left(\mathbb{Z}, \mathbb{R}^{l}\right)$.

Now, it is clear that $\sum_{t=-\infty}^{\infty} w_{2}^{\mathrm{T}}(t) w_{1}(t+n)=$ $\sum_{t=\infty}^{-\infty} w_{2}^{\mathrm{T}}(t-n) w_{1}(t)$ holds for all $w_{1}, w_{2} \in$ $l_{2}\left(\mathbb{Z}, \mathbb{R}^{\mathrm{W}}\right)$, and for all $n \in \mathbb{Z}$. Consequently, $\sum_{t=-\infty}^{\infty} v(t)^{\mathrm{T}} \Phi(M(\sigma) \ell)(t)=0$ is equivalent to $\sum_{t=-\infty}^{\infty}\left(M(\sigma)^{r \mathrm{~T}} \Phi v\right)(t)^{\mathrm{T}} \ell(t)=0$ for all $\ell \in$ $l_{2}\left(\mathbb{Z}, \mathbb{R}^{1}\right)$. This completes the proof.

\section{Dissipative- and totally dissipative systems}

In this section, we summarize the basic definitions and results about dissipative systems; a thorough exposition is given in [1]. We then define the notion of totally dissipative system, which we will characterize in several equivalent ways in Section 5 of this paper.

Let $\Phi \in \mathbb{R}^{\mathrm{w} \times \mathrm{w}}[\zeta, \eta]$, and consider a controllable behavior $\mathfrak{B} \in \mathscr{L}^{\mathrm{w}}$. $\mathfrak{B}$ is said to be dissipative with respect to $Q_{\Phi}$ if $\sum Q_{\Phi}(w) \geqslant 0$ for all $w \in \mathfrak{B} \cap$ $l_{2}\left(\mathbb{Z}, \mathbb{R}^{\mathrm{W}}\right)$. An intuitive interpretation of such definition is the following: if one considers $Q_{\Phi}$ as the rate of "energy" (i.e. $Q_{\Phi}$ is the "power") provided to the system by the environment, then $\sum Q_{\Phi}(w)$ measures the total energy exchanged with the environment while the system followed the trajectory $w$. A dissipative system is one for which the net flow of energy is nonnegative; that is, for each possible evolution $w$ the system absorbs energy from the environment.

Now assume that $\mathfrak{B}$ is controllable, with an observable image representation $w=M(\sigma) \ell$, with $M \in \mathbb{R}^{\mathrm{W} \times 1}[\xi]$. Then $\mathfrak{B}$ is dissipative w.r.t. $Q_{\Phi}$ if and only if the QdF $Q_{\Phi^{\prime}}$ induced by $\Phi^{\prime}(\zeta, \eta)=$ $M^{\mathrm{T}}(\zeta) \Phi(\zeta, \eta) M(\eta)$ satisfies

$\sum_{t=-\infty}^{\infty} Q_{\Phi^{\prime}}(\ell)(t) \geqslant 0$ for all $\ell \in l_{2}\left(\mathbb{Z}, \mathbb{R}^{1}\right)$.

It can be shown (see Proposition 3.1 of [1]) that $\sum_{t=-\infty}^{t=\infty} Q_{\Phi^{\prime}}(\ell)(t) \geqslant 0$ for all $\ell \in l_{2}\left(\mathbb{Z}, \mathbb{R}^{1}\right)$ if and only if $M\left(\mathrm{e}^{-\mathrm{i} \omega}\right)^{\mathrm{T}} \Phi\left(\mathrm{e}^{-\mathrm{i} \omega}, \mathrm{e}^{\mathrm{i} \omega}\right) M\left(\mathrm{e}^{\mathrm{i} \omega}\right) \geqslant 0$ for every $\omega \in \mathbb{R}$. Observe that in this case $M\left(\xi^{-1}\right)^{\mathrm{T}} \partial \Phi M(\xi)$ admits a symmetric factorization $M\left(\xi^{-1}\right)^{\mathrm{T}} \partial \Phi M(\xi)=$ $F^{\mathrm{T}}(-\xi) F(\xi)$, with $F \in \mathbb{R}^{1 \times 1}[\xi]$.

We now introduce the notion of storage- and of dissipation function. A $\mathrm{QdF} Q \Psi$ is said to be a storage function for $\mathfrak{B}$ with respect to $Q_{\Phi}$ if

$\nabla Q_{\Psi} \leqslant Q_{\Phi} \quad \forall w \in \mathfrak{B}$.

A QdF $Q_{\Delta}$ is said to be a dissipation function for $\mathfrak{B}$ with respect to $Q_{\Phi}$ if

$$
\begin{aligned}
Q_{\Delta}(w) \geqslant 0 \quad \forall w \in \mathfrak{B} \quad & \text { and } \\
\sum Q_{\Phi}(w) & =\sum Q_{\Delta}(w) \quad \forall w \in \mathfrak{B} \cap l_{2}\left(\mathbb{Z}, \mathbb{R}^{w}\right) .
\end{aligned}
$$

Dissipativity is characterized in terms of storage- and of dissipation functions as follows.

Proposition 3. Let $\mathfrak{B} \in \mathscr{L}^{\mathrm{w}}$ be controllable and let $\Phi \in \mathbb{R}^{\mathrm{w} \times \mathrm{w}}[\zeta, \eta]$. The following conditions are equivalent:

1. $\sum Q_{\Phi}(w) \geqslant 0$ for all $w \in \mathfrak{B} \cap l_{2}\left(\mathbb{Z}, \mathbb{R}^{\mathrm{W}}\right)$;

2. $Q_{\Phi}$ admits a storage function for $\mathfrak{B}$;

3. $Q_{\Phi}$ admits a dissipation function for $\mathfrak{B}$.

Moreover, there is a one-one correspondence between storage and dissipation functions, $Q_{\Psi}$ and $Q_{\Delta}$, respectively, defined by

$Q_{\Phi}(w)=\nabla Q_{\Psi}(w)+Q_{\Delta}(w) \quad \forall w \in \mathfrak{B}$. 
Proof. See Proposition 3.3 of [1].

If the equality $\nabla Q_{\Psi}(w)=Q_{\Phi}(w)$ holds for all $w \in$ $\mathfrak{B}$, then we call the behavior $\mathfrak{B}$ lossless with respect to $Q_{\Phi}$.

Since $\mathfrak{B}$ is controllable, it admits an observable image representation $\mathfrak{B}=\left\{w \in\left(\mathbb{R}^{\mathrm{w}}\right)^{\mathbb{Z}} \mid \exists \ell\right.$ such that $w=$ $M(\sigma) \ell$. Of course, $Q_{\Phi}(M(\sigma) \ell)=Q_{\Phi^{\prime}}(\ell)$, with

$\Phi^{\prime}(\zeta, \eta)=M^{\mathrm{T}}(\zeta) \Phi(\zeta, \eta) M(\eta)$,

the problem of studying the dissipativity or losslessness of $\mathfrak{B}$ with respect to $Q_{\Phi}$ is reduced to study the dissipativity of the $\mathrm{QdF} Q_{\Phi^{\prime}}$.

Dissipativity is a property related to the average of a functional (the dissipation rate) being nonnegative over the whole time axis. The notion of dissipativity that we now introduce, instead, is useful in the analysis of systems whose energy balance is positive on every interval- finite or infinite.

Definition 4. Let $\mathfrak{B} \in \mathscr{L}^{\mathrm{w}}$ be controllable, and let $\Phi \in \mathbb{R}^{\mathrm{W} \times \mathrm{w}}[\zeta, \eta]$ be symmetric. Let $Q_{\Delta}$ be a dissipation function for $\mathfrak{B}$ with respect to $Q_{\Phi} . \mathfrak{B}$ is called totally dissipative with respect to $Q_{\Phi}$ if for all $-\infty \leqslant t_{0} \leqslant t_{1} \leqslant+\infty$ and for all nonzero $w \in \mathfrak{B} \cap$ $l_{2}\left(\mathbb{Z}, \mathbb{R}^{\mathrm{W}}\right)$ it holds

$\sum_{k=t_{0}}^{t_{1}} Q_{\Delta}(w)(k)>0$.

The next section is devoted to the proof of a series of alternative characterizations of total dissipativeness in terms of properties of $\mathfrak{B}$ and of $Q_{\Phi}$.

\section{Main result}

The main result of this paper is the following.

Theorem 5. Let $\mathfrak{B} \in \mathscr{L}^{\mathrm{w}}$ be controllable and let $\Phi=\Phi^{\mathrm{T}} \in \mathbb{R}^{\mathrm{w} \times \mathrm{w}}$ be nonsingular. Then the following statements are equivalent:

1. $\mathfrak{B}$ is totally dissipative with respect to $\Phi$;

2. let $M \in \mathbb{R}^{\mathrm{W} \times 1}[\xi]$ induce an observable image representation of $\mathfrak{B}$. Then $M\left(\xi^{-1}\right)^{\mathrm{T}} \Phi M(\xi)$ is unimodular and such that $M\left(\mathrm{e}^{-\mathrm{i} \omega}\right)^{\mathrm{T}} \Phi M\left(\mathrm{e}^{\mathrm{i} \omega}\right) \geqslant 0$ for all $\omega \in \mathbb{R}$;
3. $\mathfrak{B} \cap \mathfrak{B}^{\perp_{\Phi}}=\{0\}$;

4. let $M \in \mathbb{R}^{\mathrm{w} \times 1}[\xi]$ induce an observable image representation of $\mathfrak{B}$. Then for every $L \in$ $\mathbb{R}^{1 \times{ }^{\mathrm{w}}}[\xi]$ left inverse of $M$, the $Q d F$ induced by $L^{\mathrm{T}}(\zeta) F^{\mathrm{T}}(\zeta) F(\eta) L(\eta) \in \mathbb{R}^{\mathrm{w} \times \mathrm{W}}[\zeta, \eta]$ induces a dissipation function for $\mathfrak{B}$ with respect to $Q_{\Phi}$, where $F \in \mathbb{R}^{1 \times 1}[\xi]$ is a unimodular spectral factor of $M\left(\xi^{-1}\right)^{\mathrm{T}} \Phi M(\xi)$;

5. there exists an image representation $w=M(\sigma) \ell$ such that the full behavior $\mathfrak{B}_{\mathrm{f}}=\{(w, \ell) \mid w=$ $M(\sigma) \ell\}$ is lossless with respect to a supply rate induced by $\operatorname{diag}\left(\Phi,-I_{1}\right)$.

Proof. We first prove the equivalence of (1) and (2).

In order to prove $(1) \Longrightarrow(2)$, taking $t_{0}=t_{1}$ in Definition 4 shows that if $\mathfrak{B}$ is totally dissipative, then any dissipation rate $Q_{\Delta}(w)$ is positive for all $w \in$ $\mathfrak{B}$. This implies also that $Q_{\Delta^{\prime}}$ is instantaneously positive, where $\Delta^{\prime}(\zeta, \eta):=M(\zeta)^{\mathrm{T}} \Delta(\zeta, \eta) M(\eta)$. Conclude from the dissipation equality (2) that

$$
\begin{aligned}
& (\zeta \eta-1) M(\zeta)^{\mathrm{T}} \Psi(\zeta, \eta) M(\eta) \\
& \quad=M(\zeta)^{\mathrm{T}} \Phi M(\eta)-\Delta^{\prime}(\zeta, \eta)
\end{aligned}
$$

holds, hence $M\left(\xi^{-1}\right)^{\mathrm{T}} \Phi M(\xi)=\Delta^{\prime}\left(\xi^{-1}, \xi\right)$. Observe that since $\mathfrak{B}$ is dissipative, $M\left(\mathrm{e}^{-\mathrm{i} \omega}\right)^{\mathrm{T}} \Phi M\left(\mathrm{e}^{\mathrm{i} \omega}\right) \geqslant 0$ for all $\omega \in \mathbb{R}$, and consequently $\Delta^{\prime}\left(\xi^{-1}, \xi\right)$ can be factored as $\Delta^{\prime}\left(\xi^{-1}, \xi\right)=D\left(\xi^{-1}\right)^{\mathrm{T}} D(\xi)$ for some $D \in \mathbb{R}^{1 \times 1}[\xi]$ (see [5, pp. 350-375]). It follows that $\Delta^{\prime}(\zeta, \eta)=D^{\mathrm{T}}(\zeta) D(\eta)$. A simple argument by contradiction yields that since $Q_{\Delta^{\prime}}$ is instantaneously positive, then $D$ must be unimodular. Consequently, $\Delta^{\prime}\left(\xi^{-1}, \xi\right)=M\left(\xi^{-1}\right)^{\mathrm{T}} \Phi M(\xi)$ is also unimodular.

The proof of (2) $\Longrightarrow(1)$ is as follows. Since $M\left(\mathrm{e}^{-\mathrm{i} \omega}\right)^{\mathrm{T}} \Phi M\left(\mathrm{e}^{\mathrm{i} \omega}\right) \geqslant 0$ for all $\omega \in \mathbb{R}$ and $M\left(\xi^{-1}\right)^{\mathrm{T}}$ $\Phi M(\xi)=\Delta^{\prime}\left(\xi^{-1}, \xi\right)$ is unimodular, $\Delta^{\prime}(\zeta, \eta)$ can be factored as $\Delta^{\prime}(\zeta, \eta)=D(\zeta)^{\mathrm{T}} D(\eta)$, with $D \in \mathbb{R}^{1 \times 1}[\xi]$ unimodular. It follows that $Q_{\Delta^{\prime}}$ is instantaneously positive, and consequently also $\sum_{k=t_{0}}^{t_{1}} Q_{\Delta}(w)(k)>0$ for all non zero $w \in \mathfrak{B}$.

In order to prove (2) $\Longleftrightarrow$ (3), consider that $M\left(\xi^{-1}\right)^{\mathrm{T}} \Phi M(\xi)$ is also unimodular if and only if $M^{r}(\xi)^{\mathrm{T}} \Phi M(\xi)$ is unimodular. From Proposition 2, observe that $M^{r}(\sigma)^{\mathrm{T}} \Phi v=0$ is a minimal kernel representation of $\mathfrak{B}^{\perp \Phi} . M^{r}(\xi)^{\mathrm{T}} \Phi M(\xi)$ being unimodular is equivalent to $M^{r}(\sigma)^{\mathrm{T}} \Phi(M(\sigma) \ell) \neq 0$ for every $\ell \neq 0$, and consequently to $M^{r}(\sigma)^{\mathrm{T}} \Phi w \neq 0$ for all $w \in \mathfrak{B}$, $w \neq 0$. This is equivalent with $\mathfrak{B} \cap \mathfrak{B}^{\perp_{\Phi}}=\{0\}$. 
We proceed to prove (2) $\Longleftrightarrow$ (4). Observe that $M\left(\mathrm{e}^{-\mathrm{i} \omega}\right)^{\mathrm{T}} \Phi M\left(\mathrm{e}^{\mathrm{i} \omega}\right) \geqslant 0$ for all $\omega \in \mathbb{R}$, if and only if there exists $F \in \mathbb{R}^{1 \times 1}[\xi]$ such that $M\left(\xi^{-1}\right)^{\mathrm{T}} \Phi M(\xi)=F\left(\xi^{-1}\right)^{\mathrm{T}} F(\xi)$. From the unimodularity of $M\left(\xi^{-1}\right)^{\mathrm{T}} \Phi M(\xi)$, it follows that $F(\xi)$ is also unimodular. Consequently, besides $w=M(\sigma) \ell$, $\mathfrak{B}$ admits another observable image representation as $w=M^{\prime}(\sigma) \ell^{\prime}$, where $M^{\prime}:=M F^{-1}$ and $\ell^{\prime}:=F(\sigma) \ell$.

Conclude from such considerations that $\mathfrak{B} \cap$ $\mathfrak{B}^{\perp_{\Phi}}=\{0\}$ if and only if $\mathfrak{B}$ is represented in observable image form as $w=M^{\prime}(\sigma) \ell^{\prime}$, with $M^{\prime}$ such that $M^{\prime}\left(\xi^{-1}\right)^{\mathrm{T}} \Phi M^{\prime}(\xi)=I_{1}$. Consequently, for every $\left(w, \ell^{\prime}\right)$ such that $w=M^{\prime}(\sigma) \ell^{\prime}$ it holds that

$\sum_{k=-\infty}^{\infty} w^{\mathrm{T}}(k) \Phi w(k)=\sum_{k=-\infty}^{\infty} \ell^{\prime \mathrm{T}}(k) \ell^{\prime}(k)$.

Now let $L \in \mathbb{R}^{1 \times \mathrm{w}}[\xi]$ be a left inverse of $M$. Consider that for every $(w, \ell)$ such that $w=M(\sigma) \ell$ it holds $L(\sigma) w=\ell$. Conclude that for every $w \in \mathfrak{B}$ of compact support

$$
\begin{aligned}
& \sum_{k=-\infty}^{\infty} w^{\mathrm{T}}(k) \Phi w(k) \\
& =\sum_{k=-\infty}^{\infty} \ell^{\prime \mathrm{T}}(k) \ell^{\prime}(k) \\
& =\sum_{k=-\infty}^{\infty}(F(\sigma) \ell)^{\mathrm{T}}(k) F(\sigma) \ell(k) \\
& =\sum_{k=-\infty}^{\infty}(F(\sigma) L(\sigma) w)^{\mathrm{T}}(k)(F(\sigma) L(\sigma) w)(k) .
\end{aligned}
$$

On the other hand, for every other left inverse $L^{\prime} \in$ $\mathbb{R}^{1 \times \mathrm{w}}[\xi]$ of $M$ it holds $L^{\prime}=L+H R$, where $H \in$ $\mathbb{R}^{(\mathrm{W}-1) \times(\mathrm{W}-1)}[\xi]$ and $R \in \mathbb{R}^{(\mathrm{W}-1) \times \mathrm{W}}[\xi]$ induces a kernel representation $R(\sigma) w=0$ of $\mathfrak{B}$. Conclude from this and from $R M=0$ that the dissipation function induced by $L^{\prime \mathrm{T}}(\zeta) L^{\prime}(\eta)$ also satisfies

$$
\begin{aligned}
& \sum_{k=-\infty}^{\infty} w^{\mathrm{T}}(k) \Phi w(k) \\
& \quad=\sum_{k=-\infty}^{\infty}(F(\sigma) L(\sigma) w)^{\mathrm{T}}(k)(F(\sigma) L(\sigma) w)(k) .
\end{aligned}
$$

This concludes the proof of the equivalence of statements (2)and (4).
Finally, we prove the equivalence of (2) and (5). The argument used in the proof of (2) and (4) allows us to conclude that there exists an observable image representation $M$ of $\mathfrak{B}$ such that $M\left(\xi^{-1}\right)^{\mathrm{T}} \Phi M(\xi)=$ $I_{\perp}$. From Proposition 1 we conclude that there exists $\Psi \in \mathbb{R}^{1 \times 1}[\zeta, \eta]$ such that $M(\zeta)^{\mathrm{T}} \Phi M(\eta)-I=(\zeta \eta-$ 1) $\Psi(\zeta, \eta)$ or equivalently, $Q_{\operatorname{diag}\left(\Phi,-I_{1}\right)}=\nabla Q \Psi$. This is equivalent with the full behavior $\mathfrak{B}_{\mathrm{f}}$ being lossless with respect to the $\mathrm{QdF}$ induced by $\operatorname{diag}\left(\Phi,-I_{1}\right)$.

Remark 6. Using the results of $[10,3]$ it is not difficult to see that under the dissipativity assumption on $\mathfrak{B}$ with respect to $\Phi$, the set $\mathfrak{B} \cap \mathfrak{B}^{\perp_{\Phi}}$ consists of all stationary trajectories of $\mathfrak{B}$ with respect to the cost functional on the external variables of $\mathfrak{B}$ associated with $\Phi$. Consequently, one additional condition equivalent to (1)-(5) of Theorem 5 is the statement

6. The set of stationary trajectories of $\mathfrak{B}$ with respect to the cost functional on the external variables induced by $\Phi$ consists of the zero trajectory only.

This formulation is close to how singular control problems are dealt with in the state-space framework. There is more than a coincidental affinity between singular control problems and totally dissipative systems; details will be provided elsewhere.

\section{Conclusions}

The main result of this paper is Theorem 5, which provides a characterization of totally dissipative systems, i.e. systems which dissipate energy for every possible transfer from one state to another. Applications of this result are being pursued in several directions, most notably in the study of singular optimal control problems, and in the development of spectral factorization algorithms for unimodular matrices.

\section{References}

[1] O. Kaneko, T. Fujii, Discrete time average positivity and spectral factorization in a behavioral framework, Systems Control Lett. 39 (1) (2000) 31-44.

[2] O. Kaneko, P. Rapisarda, Recursive exact $H_{\infty}$-identification from impulse response measurements, Systems Control Lett. 49 (5) (2003) 323-334. 
[3] M. Kuijper, Why do stabilizing controllers stabilize?, Automatica 31 (4) (1995) 621-625.

[4] J.W. Polderman, J.C. Willems, Introduction to Mathematical System Theory: A Behavioral Approach, Springer, Berlin, 1997.

[5] V.M. Popov, Hyper-Stability of Control Systems, Springer, Berlin, 1973.

[6] H.L. Trentelman, J.C. Willems, Every storage function is a state function, Systems Control Lett. 32 (1997) 249-259.

[7] J.C. Willems, Dissipative dynamical systems. part I: general theory, Arch. Rational Mech. Anal. 45 (1972) 321-351.
[8] J.C. Willems, Dissipative dynamical systems. part II: linear systems with quadratic supply rates, Arch. Rational Mech. Anal. 45 (1972) 352-393.

[9] J.C. Willems, Models for dynamics, Dynamics Reported 2 (1989) 171-269.

[10] J.C. Willems, LQ-control: a behavioral approach, in: Proceedings of the 22nd CDC, San Antonion, TX, 1993, pp. 3664-3668.

[11] J.C. Willems, H.L. Trentelman, On quadratic differential forms, SIAM J. Control Opt. 36 (5) (1998) 1703-1749. 